\title{
Galactooligosaccharides and Xylo-oligosaccharides Altering the Caecal Microbiome, Metabolome, and Transcriptome of Chickens Revealed by a Multi- Omics Analysis
}

\section{Chaowu Yang}

Sichuan Animal Sciences Academy

Mohan Qiu

Sichuan Animal Sciences Academy

Chunlin Yu

Sichuan Animal Sciences Academy

Han Peng

Sichuan Animal Sciences Academy

Xiaoyan Song

Sichuan Animal Sciences Academy

Li Yang

Sichuan Animal Sciences Academy

\section{Xia Xiong}

Sichuan Animal Sciences Academy

Chenming Hu

Sichuan Animal Sciences Academy

\section{Zengrong Zhang}

Sichuan Animal Sciences Academy

Jialei Chen

Sichuan Animal Sciences Academy

\section{Bo Xia}

Sichuan Animal Sciences Academy

Huarui Du

Sichuan Animal Sciences Academy

Qingyun Li

Sichuan Animal Sciences Academy

Xiaosong Jiang ( $\nabla$ xsjiang2017@163.com )

Sichuan Animal Sciences Academy https://orcid.org/0000-0002-7114-869X 
Research

Keywords: chickens, prebiotics, microbiota, metabolite, transcriptome

Posted Date: November 19th, 2021

DOI: https://doi.org/10.21203/rs.3.rs-1080177/v1

License: (c) (i) This work is licensed under a Creative Commons Attribution 4.0 International License. Read Full License 


\section{Abstract}

Backgroud Studies have shown that prebiotics could affect meat quality, but the underlying mechanism are poorly understood. This study aimed to investigate whether prebiotics affect chicken's meat quality through gut microbiome and metabolome.

Methods The gut content were collected from chickens fed with or without prebiotics (galactooligosaccharides or xylo-oligosaccharides) and subjected to microbiome and metabolome analyses, and chicken breast was performed transcriptome sequencing.

Results The prebiotics altered proportions of microbiota in gut contents at different levels, especially microbiota in the phylum of Bacteroidetes and Firmicutes, such as genus of Alistipes, Bacteroides, and Faecalibacterium. The prebiotics also altered contents of caecal metabolites such as lysophosphatidylcholine (lysoPC), intramuscular fat and flavor compound (Benzaldehyde and myristic acid). Differentially expressed genes (DEGs) induced by prebiotics were significantly involved in regulation of lipolysis inadipocytes and adipocytokine signaling pathway. Changes in gut microbiota and metabolites were remarkably correlated such as Bacteroidetes and Firmicutes was respectively positively and negatively correlated with lysoPC. DEGs were also interacted with caecal metabolites.

Conclusion These findings integrated and incorporated link among gut microbiota, metabolites and transcriptome, which proposed prebiotics may affect meat quality and flavor of chickens.

\section{Introduction}

The poultry is one of the fastest growing meat production, of which broiler chickens account for high population of the total poultry population in the world[1]. Over recent years, in search of building the immunocompetence of birds against infectious diseases and promoting growth, the use of antibiotics has result in the development of resistant bacteria and accumulation of antibiotic residue, which can be transferred to humans $[2,3]$. In this context, the several alternative prebiotics, including galactooligosaccharides (GOS), xylo-oligosaccharides (XOS), and fructooligosaccharides, have been demonstrated to benefit for growth and improve health of poultry $[4,5]$.

Prebiotics can be transformed into metabolites by the intestinal flora, and produce beneficial effects on performance and pathogen control of broilers [4]. Among them, GOS, an attractive food additive, has been reported to modulate the intestinal microbiota and improve intestinal development [6]. XOS, a novel prebiotic, can be utilized by probiotic strains such as Lactobacillus spp. and Bifidobacterium spp, and not degraded by recognized harmful strains [7]. In human studies, GOS and XOS consumption led to increase gut Bifidobacterium populations, increased fecal concentrations of short-chain fatty acid (SCFA) [8, 9]. Beneficial effects of the two prebiotics have also been delineated in broiler chickens, for example, the use of GOS in combination with the enzyme $\beta$-galactosidase has been also demonstrated to result in increasing abundance of Bifidobacteria and Lactobacillus [10]. Additionally, several studies have showed administration of prebiotics to chickens has been proved to have beneficial effects on broiler 
performance, intestinal health, meat quality traits [11, 12]. The juiciness, flavor and tenderness of meat quality characteristics are mainly determined by the total muscle fat content, intramuscular fat (IMF) and its fatty acid (FA) composition [13]. The development of meat flavor and aroma is mainly located in the lipid portion of the meat, while the water-soluble portion includes ingredients that contribute to develop meat flavor. Thus, prebiotics could be a positive strategy that modulates the gut microbial community and improve meat quality of broiler.

Although several studies have reported microbial regulated by prebiotics associations with broiler performance, the alteration and functional contribution of the gut microbial community in broiler chickens have not been systematically described $[14,15]$. A high interaction between the host, microbial communities and their metabolites is required for maintenance of the overall homeostasis [16-18]. Composition of gut-related metabolites can be affected by the interaction that occurred between the host, probiotics, and intestinal microflora. For example, the supplementation of multi-strain probiotics combined with $\mathrm{G}$. fructus was beneficial to the intestinal microflora composition, metabolites, and morphology in chickens [19]. Inulin and xylans (probiotics) can be actively fermented by commensal microbiota in the cecum or colon, generating bioactive metabolites represented by SCFAs, such as acetate, propionate and butyrate [20]. Additionally, the alteration of gene expression in the chicken caecum is dependence of microbiota composition [21]. Nevertheless, whether the XOS and GOS affect the metobolism in gut contents and gene expression in the chicken breast via altering the intestinal microbiota composition, and further impact meat quality is unclear.

The objective of this paper was to investigate whether prebiotics (GOS and XOS) improved meat quality of chicken through the regulation of gut microbiota structure, ceacal metabolome, and transcriptome of chicken breast. By establishing the prebiotics-induced chickens, we evaluated changes in ceacal microbiome, ceacal metabolome, and transcriptome of chicken breast in chickens fed prebiotics, and whether these alterations were correlated with each other to support the effects of prebiotics on meat quality and flavor in chickens.

\section{Materials And Methods}

\subsection{Study design and prebiotics treatments}

A total of 120 1-day-old male broiler chickens were purchased from Sichuan Daheng Poultry Breeding Company (Dayi County, Chengdu City, Sichuan Province) and were grown over a 70-day experimental period. The broiler chickens were raised at standard temperature regimens, and given feed ad libitum and had free access to water. All chickens were housed and brooded under a protocol provided by Sichuan Daheng Poultry Breeding Company.

Chickens were fed a standard corn-soybean meal based diet (Table 1). Broiler chickens were randomly allocated to 3 dietary treatments and were treated as follows: a) control group: chickens were fed a 
standard diet; b) XOS treatment group: chickens were given a standard diet and 1\% XOS; c) GOS treatment group: GOS treatment group: chickens were given a standard diet and 1\% GOS. 
Table 1

A standard corn-soybean meal based diet formula of broiler chickens.

\begin{tabular}{|c|c|c|}
\hline Composition & Diet from day 1 to 42 & Diet from day 43 to 70 \\
\hline Corn & 59.98 & 58.55 \\
\hline Soybean meal (\%) & 22.49 & 12.49 \\
\hline Rape seed cake (\%) & 2.50 & - \\
\hline Bran (\%) & - & 3.00 \\
\hline Distillers Dried Grains with Soluble & 2.50 & 4.00 \\
\hline Corn germ meal & 8.00 & 17.79 \\
\hline Soybean oil (\%) & 0.80 & 0.50 \\
\hline Limestone (Roughness) (\%) & - & - \\
\hline Limestone (\%) & 1.80 & 1.80 \\
\hline $\mathrm{CaHPO}_{4}(\%)$ & 1.00 & 1.00 \\
\hline $\mathrm{NaCl}(\%)$ & 0.30 & 0.30 \\
\hline L-Lys (\%) & 0.16 & 0.18 \\
\hline DL-Met (\%) & 0.12 & 0.07 \\
\hline Choline chloride (60\%) & 0.10 & 0.10 \\
\hline Multidimensional (\%) & 0.04 & 0.03 \\
\hline Mineral addition (\%) & 0.15 & 0.15 \\
\hline Phytase (\%) & 0.02 & 0.02 \\
\hline Compound enzymes (\%) & 0.02 & 0.02 \\
\hline Zinc bacitracin (\%) & 0.02 & - \\
\hline \multicolumn{3}{|l|}{ Nutrient composition } \\
\hline ME (MJ/kg) & 11.75 & 11.32 \\
\hline Crude protein (\%) & 18.30 & 15.30 \\
\hline Calcium (\%) & 1.00 & 0.97 \\
\hline Total phosphorus (\%) & 0.60 & 0.65 \\
\hline Non-phytic acid phosphor & 0.30 & 0.29 \\
\hline Crude fiber (\%) & 2.93 & 3.04 \\
\hline
\end{tabular}




\section{Composition $\quad$ Diet from day 1 to 42 Diet from day $\mathbf{4 3}$ to $\mathbf{7 0}$

Lysine (\%) $\quad 0.96 \quad 0.75$ \\ Methionine (\%) $\quad 0.41 \quad 0.31$ \\ 2.2. Growth monitoring and Sample collection}

The mortality and elimination rate of chickens were recorded daily, and the body weight for each group were measured once a week. The chickens were randomly selected for euthanasia. Growth performance (e.g. the breast muscle percentage, thigh muscle percentage, abdominal fat percentage and subcutaneous fat thickness) were detected and compared. The chicken breast and contents of large intestine, cecum, colon and rectum in selected broiler chickens from each group were collected, and frozen immediately at $80^{\circ} \mathrm{C}$ until used.

\subsection{S rRNA gene sequencing analysis}

Total genomic DNA from cecum, colon, and rectum contents was obtained using DNeasy PowerSoil Kit (Kurabo, Osaka, Japan) by reference to the manufacturer's recommendations. DNA quality control was checked using a NanoDropTM 2000 (Thermo Scientific, Waltham, MA, USA) spectrophotometer. The V3V4 region of bacterial 16S rRNA genes were amplified using primers 338F (5'ACTCCTRCGGGAGGCAGCAG-3') and 806 R (5' -GGACTACHVGGGTWTCTAAT-3'). Sequencing libraries were generated the TruSeq ${ }^{\circledR}$ DNA PCR-Free Sample Preparation Kit (Illumina, San Diego, USA) referring to the manufacturer's instructions. The library was submitted to paired-end sequencing $(2 \times 250$-bp read length) on the Illumina MiSeq platform (Rhonin Biotechnology Ltd, Chengdu, China).

Sequences were processed using the software package of the QIIME toolkit. Subsequently, operational taxonomic units (OTU) were picked at $97 \%$ similarity using Vsearch software. Alpha diversity index was calculated using QIIME. Beta diversity was performed on QIIME (v. 1.8.0) for Principal co-ordinates analysis (PCoA) on Bray-Curtis distance matrices. We performed linear discriminant analysis (LDA) coupled with effect size measurement (LefSe) analysis online (http://huttenhower.sph.

harvard.edu/galaxy/). Statistical analyses of the microbial relative abundances were conducted using the Kruskal-Wallis $\mathrm{H}$ test.

\subsection{Metabolic profiling of caecal contents}

Caecal contents from 30 chickens ( $\mathrm{n}=10$ per group) was utilized to extract metabolites for LC-MS analysis. Briefly, $50 \mathrm{mg}$ of the sample was transferred into $2 \mathrm{ml}$ centrifuge tube followed by the addition of a grinding ball $6 \mathrm{~mm}$ in diameter. A total of $400 \mu \mathrm{l}$ methanol (methanol: water $=4: 1(\mathrm{v}: \mathrm{v})$ ) containing $0.02 \mathrm{mg} / \mathrm{mL}$ L-2-chlorophenylalanine as internal standards was added into the sample. The mixture was ground for 6 min under a condition of $-10^{\circ} \mathrm{C}$ and $50 \mathrm{~Hz}$, and sonicated for $30 \mathrm{~min}$ at $-5^{\circ} \mathrm{C}$ and $40 \mathrm{KHz}$, and kept at $-20^{\circ} \mathrm{C}$ for $30 \mathrm{~min}$, and then centrifuged at $13000 \mathrm{~g}$ at $4^{\circ} \mathrm{C}$ for $15 \mathrm{~min}$. The supernatant was transferred into a liquid chromatography/ mass spectrometry (LC/MS) vial, and additional $20 \mu \mathrm{l}$ was used as the quality control samples for analysis. Chromatographic separation was performed in an ultra- 
performance liquid chromatography tandem fourier transform mass spectrometry UHPLC-Q Exactive system (UPLC) (Thermo Fisher Scientific, Inc. Waltham, MA, USA) using an ACQUITY UPLC HSS T3 chromatographic column ( $100 \mathrm{~mm} \times 2.1 \mathrm{~mm}$ i.d., $1.8 \mu \mathrm{m}$; Waters, Milford,USA) with a constant column temperature of $40^{\circ} \mathrm{C}$. After equilibration for $2 \mathrm{~min}$, the column flow rate remained at $0.40 \mathrm{~mL} / \mathrm{min}$, and the injection volume of each sample was $2 \mu \mathrm{l}$. The mobile phase consisted of solvent $\mathrm{A}\left(\mathrm{H}_{2} \mathrm{O}+0.1 \%\right.$ formic acid) and solvent $B$ (acetonitrile: isopropanol $=1: 1(\mathrm{v}: \mathrm{v})+0.1 \%$ formic acid). The MS experiments were respectively performed with spray voltages of $3500 \mathrm{~V}$ and $2800 \mathrm{~V}$ in positive and negative modes. Sheath gas and auxiliary gas were respectively at 40 and 10 arbitrary units. The heater temperature and capillary temperature was $400^{\circ} \mathrm{C}$ and $320^{\circ} \mathrm{C}$, respectively. The voltages of the S-Lens were $50 \mathrm{~V}$ in positive and negative modes. The range of Orbitrap analyzer scan type was $70-1050 \mathrm{~m} / \mathrm{z}$ for full scan at a resolution of 70000 . The normalized collision energy was $204060 \mathrm{eV}$.

The raw data was processed by Progenesis QI (Waters Corporation, Milford, USA). The main procedure consisted of integration, normalization and alignment of peak intensities, and a list of $\mathrm{m} / \mathrm{z}$ and retention time with corresponding intensities was obtained for all metabolites in per sample. Subsequently, the data was mapped into the Human Metabolome Database (HMDB, http://www.hmdb.ca/) and the Metabolite and Tandem MS Database (METLIN, https://metlin.scripps.edu/) to obtain metabolites. We obtain different metabolites through multidimensional analysis and one-dimensional analysis. Metabolites with the variable importance $(\mathrm{VIP}) \geq 1.0$ and $\mathrm{P} \leq 0.05$ were considered as different metabolites. The normalized data were utilized to conduct principal component analysis (PCA), partial least-squares discriminant analysis (PLS-DA), orthogonal to partial least squares-discriminate analysis (OPLS-DA), and permutation testing.

\subsection{Transcriptome sequencing analysis}

Total RNA from chicken breast ( $\mathrm{n}=4$ per group) was isolated using TRIzol reagent (Invitrogen, 15596-018). After removing genomic DNA contaminations and assessing RNA integrity, the quality and quantity of RNA were evaluated using an Agilent 2100 bioanalyzer with the RNA 6000 Nano Chip (Agilent Technologies) and Agilent 2100 Bioanalyzer (Agilent Technologies, CA, USA). The sequencing library was generated from the NEB Next UltraTM RNA Library prep Kit for Illumina (NEB, USA) according to the instruction manual. The twelve cDNA libraries that were obtained were sequenced on Hi-Seq platform (Illumina, USA) and generated paired-end reads.

The Fast-QC (http://www.bioinformatics.babraham.ac.uk/projects/fastqc/) was used to analyze the raw data to filter away the low-quality sequences and adaptor sequences. Then, we matched the high quality clean reads obtained to the Ensembl Gallus_gallus-5.0 (Gallus_gallus. Gallus_gallus-5.0.dna.toplevel.fa) database. The level of gene expression in chicken breast was calculated by FPKM, the differentially expressed genes (DEGs) with a $P$ value $<0.05$ and | log2 (fold change) $\mid>1$ was determined by the DESeq2 algorithm. Function analysis of DEGs were performed using Gene ontology (GO, http: \\www.geneontology.org \), and pathway analysis were assessed by kyoto encyclopedia of genes and genomes (KEGG, http:\\www.genome.jp\kegg\analyses). 


\subsection{Statistical analyses}

All data were analyzed with Graph Pad Prism 8 software. Growth performance parameter were presented as mean \pm standard deviation and analyzed through $\mathrm{t}$ test. All results were considered statistically significant at $P<0.05$.

\section{Results}

\subsection{Prebiotic supplementation slightly improves growth performance of broiler chickens}

As depicted in Table 2, the GOS had a growth promoting effect on the average body weight of chickens, while there were no significant differences between $X O S$ treatment group and control group $(P>0.05)$. No statistical differences in performance metric of feed conversion ratio. Additionally, the breast muscle percentage and thigh muscle percentage were slightly increased in prebiotics group relative to the control group $(P>0.05)$. The abdominal fat percentage and subcutaneous fat thickness $(\mathrm{cm})$ showed slight decrease in prebiotic treatment group $(P>0.05)$. Feed conversion ratio of the prebiotic treatment group was lower that of control group $(P>0.05)$. Thus, considering the growth performance, the prebiotic group was slightly superior to the control group.

Table 2

Growth and meat production of 70-day-age chickens in different prebiotics-treated groups.

\begin{tabular}{|llll|}
\hline Performance Metric & Control group $(\mathbf{n = 1 0})$ & XOS group $(\mathbf{n = 1 0})$ & GOS group $(\mathbf{n = 9})$ \\
\hline Average body weight (g) & $1768 \pm 163$ & $1773 \pm 165$ & $1837 \pm 171$ \\
\hline Breast muscle percentage (\%) & $15.66 \pm 1.00$ & $16.03 \pm 1.29$ & $16.43 \pm 1.96$ \\
\hline Thigh muscle percentage (\%) & $19.31 \pm 0.94$ & $19.87 \pm 1.03$ & $20.02 \pm 6.36$ \\
\hline Abdominal fat percentage (\%) & $3.26 \pm 1.5$ & $3.59 \pm 1.3$ & $3.35 \pm 1.2$ \\
\hline Subcutaneous fat thickness (cm) & 3.75 & 3.38 & 3.61 \\
\hline Feed conversion ratio & $2.4: 1$ & $2.35: 1$ & $2.30: 1$ \\
\hline Morbidity and mortality cases & $5.2 \%$ & $5.0 \%$ & $4.3 \%$ \\
\hline Notes: There are no significant difference between the prebiotics group and the control group. \\
\hline
\end{tabular}

\subsection{Prebiotic intervention alters microbiome composition}

In our present microbiome investigation, the caecal microbial communities were compared between prebiotics (GOS and XOS) treatment group and control group. A total number of 1513565 valid reads and 
795 OTUs at $97 \%$ sequence similarity were obtained from all samples, respectively. The rank abundance analysis on the OUT level revealed that the richness and evenness of the microbiota composition was the similar among three groups (Figure 1A). The extent of the similarity of gut microbial communities between the three groups was measured PCoA at the OTU level, the results showed that the gut microbial communities was significantly separated between GOS, XOS and control group (Figure 1B). Moreover, we found that the diversity of the microbial community significantly decreased in the prebiotics treatment group, especially in XOS group, as showed by abundance-based coverage estimators (ACE) and Chao1 indices (Figure 1C and 1D).

Then, we displayed the composition of caecal microbiota at family and genus levels. Microbiota of the 30 samples from the prebiotics treatment group and control group were altered at family level. As shown in Figure 1E, the abundance of Ruminococcaceae, Barnesiellaceae, and Acidaminococcaceae were increased in the prebiotics treatment group. Unlike these bacteria, the prebiotics treatment group had a lower average relative abundance of Bacteroidaceae and Lactobacillaceae compared with control (Figure $1 \mathrm{E})$. At the Top10 genus level, the increased proportions of Alistipes, Faecalibacterium, unclassified_f_Barnesiellaceae, and Phascolarctobacterium, decreased proportions of Bacteroides and Lactobacillus were observed in prebiotics treatment group in comparison to control (Figure 1F).

Consistent with above results, the circos plot at genus level revealed that Alistipes was the dominant flora in GOS group, accounting for $19.86 \%$ (Figure 2A). Faecalibacterium and Bacteroides were the dominant flora in XOS group, accounting for $18.15 \%$ and $17.73 \%$, respectively (Figure $2 \mathrm{~A}$ ). Bacteroides was the only dominant flora in control group, accounting for about 20.82\% (Figure 2A). Importantly, Alistipes and Bacteroides are a genus in the phylum Bacteroidetes, and Faecalibacterium is a genus in the phylum Firmicutes, indicating that prebiotics treatment may affect the quality of chicken mainly by changing the composition of Bacteroidetes and Firmicutes. Subsequently, LEfSe analysis revealed specific bacteria that were associated with prebiotics (Figure $2 B$ ). Several microbiota including $f_{-}$ Porphyromonadaceae_g_Barnesiella_0TU436, f_Bacteroidaceae_g_Bacteroides_0TU370, and s_Bacteroides_caecigallinarum were all significantly over-represented (all LDA scores $(\log 10)>3$ ) in the feces of chickens fed with XOS. Interestingly, the Porphyromonadaceae, Bacteroidaceae, and Bacteroides are belong to the phylum Bacteroidetes. The c_Clostridia_O_Oscillospirales_OTU445, belong to Firmicutes phylum, was identified as specific taxa in chickens treated with GOS (all LDA scores $(\log 10)>2$ ). Therefore, these results again suggested that prebiotics treatment primarily altered the abundance of Bacteroidetes and Firmicutes.

\subsection{Prebiotic changes global metabolome of caecum}

Since signature of microbiota related with prebiotics in the chickens was demonstrated, we speculated that alterations in metabolic pathways may be at least partially influenced by prebiotic-driven gut microbiota in chickens. Thus, we subsequently analyzed intestinal metabolites in caecal feces of chickens, using a nontargeted LC-MS technology. As shown in Fig. S1A-1D, the score plots of PLS-DA confirmed that the metabolic profiles had significant differences between prebiotics treatment group and control. Permutation test for the OPLS-DA model to further visualized group separation that XOS group 
and control group generated intercepts of R2 $=0.9848$ and Q2 $=-0.1713$ (Fig. S1E), and that of GOS group and $\mathrm{E}$ group produced intercepts of R2 $=0.9851$ and Q2 $=-0.1205$ (Fig. S1F), which revealed OPLS-DA had well fitted-effect.

Subsequently, we summarized the distribution of differential metabolites separating the two groups. Overall, 135 differential metabolites were identified in comparison of prebiotics treatment group relative to control (VIP > 1, P < 0.05). There were 79 and 92 differential metabolites respectively found in XOS $v s$ control and GOS vs control, respectively (Table S1 and Table S2). A heat map was utilized to visualize the abundance of the differential metabolites (Figure 3A and 3B). In total, two clusters were respectively generated in XOS vs control and GOS vs control. Among these, 36 metabolites were shared (Table S3), including seven type of lysophosphatidylcholine (LysoPC), such as PC(16:0/0:0)[U], LysoPC(16:1(9Z)/0:0), LysoPC(18:1(11Z)), LysoPC(16:1(9Z)), LysoPC(18:1(9Z)), LysoPC(16:0), and LysoPC(P-16:0), and two metabolites of the shikimic acid pathway (such as shikimic acid, cinnamic acid). Together, these pieces of evidence indicated that production of intestinal metabolites regulated by different prebiotic, especially LysoPC and shikimic acid pathway.

Moreover, we identified several altering metabolic pathways that specific related with prebiotics treatment. As depicted in Figure 3C and 3D, the Phenylalanine, tyrosine and tryptophan biosynthesis, Purine metabolism, and Glutathione metabolism were observed to be strikingly disturbed in response to XOS. Interestingly, the biosynthesis of these amino acids depend on shikimic acid pathway. The perturbations of D-Glutamine and D-glutamate metabolism, Glutathione metabolism and Glycerophospholipid metabolism (the enriched pathway of LysoPC -like metabolites) were discovered in response to GOS treatment. These results suggested that prebiotics feeding may induce changes in intestinal microbial LysoPC and shikimic acid metabolism in broilers chicken.

\subsection{Alteration of meat metabolites}

Subsequently, we further detected the concentration of common nutrients, including the proteins, amino acids, fatty acids in the chicken breast. As presented in Table S4, overall, prebiotics treatment significantly decreased the content of histidine compared to control samples but had no significant effect on other amino acids in chicken breast. Although administration of prebiotics significantly decreased the proportion of some fatty acids, such as myristic acid (C14) and palmitoleic acid (C16), the proportion of IMF remarkably increased in prebiotics group. Notably, although concentration of these metabolites such as oleic acid and polyunsaturated fatty acids (linoleic acid and arachidonic acid) had no significant alteration, that of them had a slight increase in chicken breast and viscera samples from XOS or GOS treatment group relative to control group. Accumulating studies have confirmed that juiciness, flavor, and tenderness correlate positively with the muscle total fat content $[22,23]$. Polyunsaturated fatty acids are heated and oxidized to generate volatile components, such as 2, 4-decaldehyde, which improve the flavor of meat [24]. Together, the addition of prebiotics had an effect on meat quality and flavor of chicken. 


\subsection{Profiling of genes expressed in the chicken breast of the prebiotics treatment group and control group}

We further investigated the metabolite gene response. In total, 599 genes that were significantly altered by transcriptome sequencing identified. There were 381 and 298 DEGs genes respectively obtained in XOS vs control and GOS vs control (Table S5). Among these, 80 DEGs were shared, including 36 upregulated DEGs and 42 down-regulated DEGs (data not shown). We further analyzed the expression tendency of these common genes, the trend for 10 genes of subcluster_5, such as LRRTM3, LRRC10B, and ENSGALG00000046353 was similar; these genes all highly expressed in prebiotics group relative to control group. The trend for 4 genes of subcluster_9, such as CFAP44 and CCL21 presented same result as that for subcluster_5 (Figure 4A). Subsequently, corresponding functions of the 80 common genes were determined (Figure 4B), which were mainly found to function as biological process in the "regulation of lipid kinase activity", "regulation of lipid metabolic process", "regulation of phospholipid metabolic process", and "regulation of hormone biosynthetic process. KEGG annotation analysis showed these genes were mainly involved in organismal systems of "endocrine system", "immune system", "immune system", "digestive system", and various metabolism (e.g. nucleotide, carbohydrate, biosynthesis of other secondary, amino acid metabolism) (Figure 5A). Subsequently, KEGG enrichment analysis delineated 80 genes primarily implicated in metabolism pathway of "regulationof lipolysis inadipocytes", "adipocytokine signaling pathway", "protein digestion and absorption", "cytokine-cytokine receptor interaction" and "Jak-STAT signaling pathway" (Figure 5B). These pathways were related with lipid and protein metabolism and may affect meat quality and flavor.

\subsection{Caecal microbiota correlated to growth performance of broiler chickens}

To investigate the correlation between caecal microbiota alteration and growth performance, we analyzed the correlation between species abundance (Bacteroidetes and Firmicutes, and other taxa at family level mentioned above) and growth performance by Pearson correlation coefficient analysis. At the phylum level, only Bacteroidetes showed significantly positively correlation with average body weight (Figure 6). At the family level, caecal microbiota of Barnesiellaceae and Ruminococcaceae were significantly positively correlation with breast muscle percentage and average body weight, respectively (Figure 6). No significant correlation was detected between other caecal microbiota and growth performance.

\subsection{Correlations between the prebiotics-induced gut microbiome and metabolome}

A pearman correlation analysis of differential metabolites and top40 OTUs in abundance (microbes) was performed. As depicted in Figure 7A, based on differential metabolites in XOS group, the f_Acidaminococcaceae_g_Phascolarctobacterium_0TU754 was significantly positively related to retapamulin, N-Methyl-14-O-demethylepiporphyroxine, mandelonitrile rutinoside, N-Arachidonoyl tyrosine, benzaldehyde and L-Tryptophan. 
F_Rikenellaceae_g_Alistipes_OTU714 and F_Rikenellaceae_g_Alistipes_OTU326 were significantly positively correlated with LysoPC such as LysoPC (P-16:0), LysoPC (16:0).

F_Lactobacillaceae_g_Lactobacillus_OTU4 was remarkably negatively related to mandelonitrile rutinoside, alliosterol 1-rhamnoside 16-galactoside, halocins, benzaldehyde and 3-Buten-1-amine. The indoleacetic acid and hypoxanthine were also found to be dramatically negatively related with f_Lachnospiraceae_g_CHKCI001_0TU416.

Based on differential metabolites in GOS group (Figure S2), F_Rikenellacea_g_Alistipes_OTU756 was closely positively correlated with cucurbic acid, cerebronic acid, 3"-0-Caffeoylcosmosiin and choline except for LysoPC. The F_Rikenellacea_g_Alistipes_OTU714, f_Barnesiellaceae_g_Barnesiella_OUT78, and f_Barnesiellaceae_g_Barnesiella_OTU155 were positively correlated with shikimic acid. The 1, 25Dihydroxyvitamin D3-26, 23-lactone and choline were found to present remarkably positive and negative correlation with f_Barnesiellaceae_g_Barnesiella_OTU436, respectively. L-Glutamate was dramatically positively associated with $\mathrm{f} \_$Barnesiellaceae_g_unclassified_f_OTU716. Together, these results revealed above dominant microbiota caused the differences in gut metabolites in the chickens fed with prebiotics.

Moreover, we also investigate the correlation between species abundance (Bacteroidetes and Firmicutes) and seven type of LysoPC by Pearson correlation coefficient analysis. As depicted in Figure 8A-8B, Bacteroidetes was significantly positive correlated with LysoPC(18:1(11Z)) and LysoPC(16:0). While Firmicutes was significantly negative correlated with LysoPC(16:0), LysoPC(16:1(9Z)/0:0), LysoPC(P16:0), LysoPC(16:1(9Z)), and LysoPC(18:1(9Z)) (Figure 8C-8G). These results suggest that the change of caecal microflora Bacteroidetes and Firmicutes are related to the metabolism of differential metabolite LysoPC.

\subsection{Metabolite-gene network analysis}

To extract the interactions among the all differential metabolites and gene expression in prebioticssupplemented chickens, a network diagram was constructed. Among the differential metabolites, a total of twelve metabolites interacted with differentially expressed genes (Figure 7B). Particularly, the AMD1(Sadenosylmethionine decarboxylase)-cadaverine or omithine pairs, and PLA2G1B (phospholipase A2 group 1B) - indoleacetic acid pairs were discovered in the network. These genes were likely hubs of the prebiotics treatment on meat quality of chickens.

\section{Discussion}

Growing evidence shows that novel additives such as prebiotics or probiotics can regulate composition and/or activity of the gut microbiota, thus attributing a beneficial physiological effect on the host $[25,26]$. Beneficial effects of prebiotics (XOS and GOS) on performance and gut microbiota have already been demonstrated in chickens $[10,27,28]$. However, whether the gut microbial community, metabolites, and transcriptomic changes of chicken breast were correlated in chicken fed with prebiotics is not unknown. In the study, we proved the clue that prebiotics improved meat quality and flavor in broiler through modulating gut microbiota, metabolites and chicken breast transcriptome. 
Selective fermentation of some prebiotics has been demonstrated to cause alteration in the composition and/or activity of the gastrointestinal microbiota, contributing to the host health [29]. A study has reported dietary xylooligosaccharides prebiotics can improve growth performance, enhance endocrine metabolism and immune function of broilers [30]. Additionally, changes in enteric bacteria in the cecum [31] and improved intestinal morphology have been found in chickens given with dietary mannanoligosaccharide [32]. In the present study, supplementation with either GOS or XOS improved the growth performance of chickens, and increased the relative abundance of dominant and beneficial bacteria (Alistipes, Faecalibacterium, Phascolarctobacterium), while decreased that of potential bacterial pathogens (Rikenellaceae_RC9_gut_group). It can be also assumed that the improvement in growth performance is associated with the beneficial effect of prebiotics on the intestinal microbiota.

LysoPC, a derivative of PC hydrolyzed by phospholipase $A 2$, is a highly abundant bioactive lipid mediator that exist in circulation [33]. Different contens of PC determine whether the vesicles release cholesterol, so the changes in LysoPC are a dynamic reflection of cholesterol [33]. In this study, prebiotic treatment resulted in significant changes in the content of 7 types of PC, suggesting that prebiotic feeding disrupted the original cholesterol metabolism of broiler chicken. Furthermore, we found that caecal microflora Bacteroidetes and Firmicutes are related to the metabolism of LysoPC. Consistent with our results, Bai et al. reported that the inflammation-related metabolites of LysoPC(16:0) was significantly correlated with genera that belonged to phyla Firmicutes [34]. The results of Gao et al. reveal that LysoPC(20:0) has a similar correlation trend with us, LysoPC(20:0) was respectively negatively correlated with Firmicutes and positively correlated with Bacteroidetes [35]. Therefore, we speculated that prebiotic feeding stimulated the changes of Firmicutes and Bacteroidetes, which further changed the content of LysoPC and affected lipid accumulation in broiler chicken.

The saturated fatty acids and omega ( $\omega$ )-3 polyunsaturated fatty acids (PUFAs) like linoleic acid and alinolenic acid, and IMF were also associated with the umami flavor [36]. Similarly, the concentration of flavoring fatty acids like myristic acid and IMF were significantly higher in chickens fed with prebiotics. The concentration of PUFAs has a slight increase in prebiotics group. The fatty acids play an important role in determining the meat quality and flavor of chickens. For example, the myristic acid (C14:0) contribute to flavor and is used as a flavoring agent in food items [37]. The benzaldehyde is known to arise from linolenic acid (C18:3n-6), and considered as the characteristic flavor components [38]. Here, the higher concentration of benzaldehyde was found in prebiotics group, and it had significantly positive and negative correlation with g_Phascolarctobacterium_OTU754 and g_Lactobacillus_OTU4, respectively. Consistently, the abundance of Phascolarctobacterium and Lactobacillus respectively increased and decreased in prebiotics group in comparison to control. Other non-volatile flavor precursor components including amino acids also affect the meat quality of meat [39]. The monosodium glutamate-like amino acids, including glutamic and aspartic acid were ingredients for umami taste [40]. Here, the L-Glutamate was found to be dramatically positively related with $\mathrm{f} \_$Barnesiellaceae_g_unclassified_f_OTU716; the proportion of Barnesiellaceae increased in prebiotics group. Additionally, other differential metabolites (e.g. retapamulin, N-Methyl-14-O-demethylepiporphyroxine, LysoPC, et al) had also correlation with microbial taxa (e.g. g_Alistipes_OTU714 and OTU326, g_CHKCI001_OTU416, g_Barnesiella_OTU436, et 
al). Therefore, the microbiota -metabolites axis was regulated by prebiotics may affect meat quality and flavor of chickens.

The fatty acids are oxidized and used as energy source, or stored and deposited in adipose tissues [41].

The dietary fatty acids exert regulator role in gene expression and finally control enzyme activity [42].

Therefore, the lipid uptake, transport, storage and biosynthesis are complex steps to regulate balance of lipid metabolism. We found the perturbations of lipid metabolism related pathway such as D-Glutamine and D-glutamate metabolism, Glutathione metabolism and Glycerophospholipid metabolism were discovered in response to prebiotics treatment. To further understand the potential mechanism of lipid metabolism, the GO was analyzed. Many DEGs in chicken breast from prebiotics group and control group were mainly involved in biological process of the "regulation of lipid kinase activity" and "regulation of lipid. To further study the links between gut metabolites and gene expression, a network diagram was constructed. The twelve metabolites interacted with many genes (e.g. AMD1 and PLA2G1B).

Accumulating studies have showed AMD1 is associated with sustaining polyamine metabolism in prostate cancer [43, 44]. PLA2G1B, as a secreted phospholipase, is reported to mediate lipid absorption [45]. However, the links of these genes with metabolism were further confirmed.

\section{Conclusion}

The beneficial effect of prebiotics on gut microbiome was determined. The prebiotics affected complicated interactions, leading to alterations in the gut microbiome, metabolites, and gene expression in chickens and further contribute to regulate the meat quality and flavor on chicken.

\section{Abbreviations}

galactooligosaccharides (GOS), xylo-oligosaccharides (XOS), short-chain fatty acid (SCFA), intramuscular fat (IMF), fatty acid (FA), operational taxonomic units (OTU), Principal co-ordinates analysis (PCoA), linear discriminant analysis (LDA) coupled with effect size measurement (LefSe), principal component analysis (PCA), partial least-squares discriminant analysis (PLS-DA), orthogonal to partial least squaresdiscriminate analysis (OPLS-DA), Differentially expressed genes (DEGs), lysophosphatidylcholine (LysoPC), myristic acid (C14), palmitoleic acid (C16), polyunsaturated fatty acids (PUFAs)

\section{Declarations}

\section{Ethics approval and consent to participate}

All experimental protocols were approved by the animal care and ethical committee of Sichuan Animal Science Academy.

\section{Consent for publication}

Not applicable 
Availability of data and materials

All data generated or analysed during this study are included in this published article and its supplementary information files.

\section{Competing interests}

The authors declare no conflict of interest.

\section{Funding}

This work was supported by National Modern Agricultural Industrial Technology System Construction Project (No. CARS-41-G04); and The Sichuan Science and Technology Program (No. 2020YFN0146, 2021YFYZ0031, 2021YFYZ0009 and 2021YFN0029).

\section{Author contributions}

Conceptualization, XS.J. and MH.Q; Data curation, H.P. and XY.S.; Formal analysis, L.Y., X.X. and CM.H.; Methodology, ZR.Z. and JL.C.; Project administration, B.X.; Resources, XS.J.; Software, HR.D; Validation, QY.L.; Writing Review \& editing, CL.Y., CW.Y., XS.J. and MH.Q. All authors have read and agreed to the published version of the manuscript.

Acknowledgments: Not applicable.

\section{References}

1. Yadav $S$ and Jha R. Strategies to modulate the intestinal microbiota and their effects on nutrient utilization, performance, and health of poultry. J Anim Sci Biotechnol. 2019;10:2. doi: 10.1186/s40104-018-0310-9.

2. Sweeney MT, Lubbers BV, Schwarz S and Watts JL. Applying definitions for multidrug resistance, extensive drug resistance and pandrug resistance to clinically significant livestock and companion animal bacterial pathogens-authors' response. J Antimicrob Chemother. 2019;74(2): 536-537. doi: 10.1093/jac/dky470.

3. Costa T, Linhares I, Ferreira R, Neves $\mathrm{J}$ and Almeida A. Frequency and Antibiotic Resistance of Bacteria Implicated in Community Urinary Tract Infections in North Aveiro Between 2011 and 2014. Microb Drug Resist. 2018;24(4): 493-504. doi: 10.1089/mdr.2016.0318.

4. Kim GB, Seo YM, Kim CH and Paik IK. Effect of dietary prebiotic supplementation on the performance, intestinal microflora, and immune response of broilers. Poult Sci. 2011;90(1): 75-82. doi: 10.3382/ps.2010-00732.

5. Huyghebaert G, Ducatelle R and Van Immerseel F. An update on alternatives to antimicrobial growth promoters for broilers. Vet J. 2011;187(2): 182-8. doi: 10.1016/j.tvjl.2010.03.003. 
6. Boehm $G$ and Moro G. Structural and functional aspects of prebiotics used in infant nutrition. $J$ Nutr. 2008;138(9): 1818S-1828S. doi: 10.1093/jn/138.9.1818S.

7. Kondepudi KK, Ambalam P, Nilsson I, Wadstrom T and Ljungh A. Prebiotic-non-digestible oligosaccharides preference of probiotic bifidobacteria and antimicrobial activity against Clostridium difficile. Anaerobe. 2012;18(5): 489-97. doi: 10.1016/j.anaerobe.2012.08.005.

8. Tateyama I, Hashii K, Johno I, lino T, Hirai K, Suwa Y, et al. Effect of xylooligosaccharide intake on severe constipation in pregnant women. J Nutr Sci Vitaminol (Tokyo). 2005;51(6): 445-8. doi: 10.3177/jnsv.51.445.

9. Sawicki CM, Livingston KA, Obin M, Roberts SB, Chung M and McKeown NM. Dietary Fiber and the Human Gut Microbiota: Application of Evidence Mapping Methodology. Nutrients. 2017;9(2). doi: 10.3390/nu9020125.

10. Jung SJ, Houde R, Baurhoo B, Zhao X and Lee BH. Effects of galacto-oligosaccharides and a Bifidobacteria lactis-based probiotic strain on the growth performance and fecal microflora of broiler chickens. Poult Sci. 2008;87(9): 1694-9. doi: 10.3382/ps.2007-00489.

11. Tavaniello S, Slawinska A, Prioriello D, Petrecca V, Bertocchi M, Zampiga M, et al. Effect of galactooligosaccharides delivered in ovo on meat quality traits of broiler chickens exposed to heat stress. Poult Sci. 2020;99(1): 612-619. doi: 10.3382/ps/pez556.

12. Tavaniello S, Maiorano G, Stadnicka K, Mucci R, Bogucka J and Bednarczyk M. Prebiotics offered to broiler chicken exert positive effect on meat quality traits irrespective of delivery route. Poult Sci. 2018;97(8): 2979-2987. doi: 10.3382/ps/pey149.

13. Hocquette JF, Gondret F, Baeza E, Medale F, Jurie C and Pethick DW. Intramuscular fat content in meat-producing animals: development, genetic and nutritional control, and identification of putative markers. Animal. 2010;4(2): 303-19. doi: 10.1017/S1751731109991091.

14. Wu X, Yang P, Sifa D and Wen Z. Effect of dietary stevioside supplementation on growth performance, nutrient digestibility, serum parameters, and intestinal microflora in broilers. Food Funct. 2019;10(5): 2340-2346. doi: 10.1039/c8fo01883a.

15. Richards PJ, Flaujac Lafontaine GM, Connerton PL, Liang L, Asiani K, Fish NM, et al. GalactoOligosaccharides Modulate the Juvenile Gut Microbiome and Innate Immunity To Improve Broiler Chicken Performance. mSystems. 2020;5(1). doi: 10.1128/mSystems.00827-19.

16. Rooks MG and Garrett WS. Gut microbiota, metabolites and host immunity. Nat Rev Immunol. 2016;16(6): 341-52. doi: 10.1038/nri.2016.42.

17. Alrubaye B, Abraha M, Almansour A, Bansal M, Wang H, Kwon YM, et al. Microbial metabolite deoxycholic acid shapes microbiota against Campylobacter jejuni chicken colonization. PLoS One. 2019;14(7): e0214705. doi: 10.1371/journal.pone.0214705.

18. Ren H, Vahjen W, Dadi T, Saliu EM, Boroojeni FG and Zentek J. Synergistic Effects of Probiotics and Phytobiotics on the Intestinal Microbiota in Young Broiler Chicken. Microorganisms. 2019;7(12). doi: 10.3390/microorganisms7120684. 
19. Chang CH, Teng PY, Lee TT and Yu B. Effects of Multi-Strain Probiotics Combined with Gardeniae fructus on Intestinal Microbiota, Metabolites, and Morphology in Broilers. J Poult Sci. 2019;56(1): 3243. doi: $10.2141 /$ jpsa.0170179.

20. Franzenburg S, Walter J, Kunzel S, Wang J, Baines JF, Bosch TC, et al. Distinct antimicrobial peptide expression determines host species-specific bacterial associations. Proc Natl Acad Sci U S A. 2013;110(39): E3730-8. doi: 10.1073/pnas.1304960110.

21. Wang H, Li S, Fang S, Yang $X$ and Feng J. Betaine Improves Intestinal Functions by Enhancing Digestive Enzymes, Ameliorating Intestinal Morphology, and Enriching Intestinal Microbiota in Highsalt stressed Rats. Nutrients. 2018;10(7). doi: 10.3390/nu10070907.

22. Chartrin P, Meteau K, Juin H, Bernadet MD, Guy G, Larzul C, et al. Effects of intramuscular fat levels on sensory characteristics of duck breast meat. Poult Sci. 2006;85(5): 914-22. doi:

10.1093/ps/85.5.914.

23. Liu L, Cui H, Xing S, Zhao G and Wen J. Effect of Divergent Selection for Intramuscular Fat Content on Muscle Lipid Metabolism in Chickens. Animals (Basel). 2019;10(1). doi: 10.3390/ani10010004.

24. Calkins CR and Hodgen JM. A fresh look at meat flavor. Meat Sci. 2007;77(1): 63-80. doi: 10.1016/j.meatsci.2007.04.016.

25. da Silva TF, Casarotti SN, de Oliveira GLV and Penna ALB. The impact of probiotics, prebiotics, and synbiotics on the biochemical, clinical, and immunological markers, as well as on the gut microbiota of obese hosts. Crit Rev Food Sci Nutr. 2021;61(2): 337-355. doi: 10.1080/10408398.2020.1733483.

26. Gill SK, Rossi M, Bajka B and Whelan K. Dietary fibre in gastrointestinal health and disease. Nat Rev Gastroenterol Hepatol. 2021;18(2): 101-116. doi: 10.1038/s41575-020-00375-4.

27. De Maesschalck C, Eeckhaut V, Maertens L, De Lange L, Marchal L, Nezer C, et al. Effects of XyloOligosaccharides on Broiler Chicken Performance and Microbiota. Appl Environ Microbiol. 2015;81(17): 5880-8. doi: 10.1128/AEM.01616-15.

28. Jiang S, Yan FF, Hu JY, Mohammed A and Cheng HW. Bacillus subtilis-Based Probiotic Improves Skeletal Health and Immunity in Broiler Chickens Exposed to Heat Stress. Animals (Basel). 2021;11(6). doi: 10.3390/ani11061494.

29. Shah BR, Li B, Al Sabbah H, Xu W and Mraz J. Effects of prebiotic dietary fibers and probiotics on human health: With special focus on recent advancement in their encapsulated formulations. Trends Food Sci Technol. 2020;102:178-192. doi: 10.1016/j.tifs.2020.06.010.

30. Zhenping S, Wenting L, Ruikui Y, Jia L, Honghong L, Wei S, et al. Effect of a straw-derived xylooligosaccharide on broiler growth performance, endocrine metabolism, and immune response. Can J Vet Res. 2013;77(2): 105-9.

31. Spring P, Wenk C, Dawson KA and Newman KE. The effects of dietary mannaoligosaccharides on cecal parameters and the concentrations of enteric bacteria in the ceca of salmonella-challenged broiler chicks. Poult Sci. 2000;79(2): 205-11. doi: 10.1093/ps/79.2.205.

32. Baurhoo B, Ferket PR and Zhao X. Effects of diets containing different concentrations of mannanoligosaccharide or antibiotics on growth performance, intestinal development, cecal and 
litter microbial populations, and carcass parameters of broilers. Poult Sci. 2009;88(11): 2262-72. doi: 10.3382/ps.2008-00562.

33. Tazuma S, Kanno K, Sugiyama A and Kishikawa N. Nutritional factors (nutritional aspects) in biliary disorders: bile acid and lipid metabolism in gallstone diseases and pancreaticobiliary maljunction. J Gastroenterol Hepatol. 2013;28 (4): 103-107. doi: 10.1111/jgh.12241.

34. Bai S, Xie J, Bai H, Tian T, Zou T and Chen JJ. Gut Microbiota-Derived Inflammation-Related Serum Metabolites as Potential Biomarkers for Major Depressive Disorder. J Inflamm Res. 2021;14: 37553766. doi: $10.2147 / J I R . S 324922$.

35. Gao Y, Liu Y, Ma F, Sun M, Song Y, Xu D, et al. Lactobacillus plantarum Y44 alleviates oxidative stress by regulating gut microbiota and colonic barrier function in Balb/C mice with subcutaneous $\mathrm{d}$ galactose injection. Food Funct. 2021;12(1): 373-386. doi: 10.1039/d0fo02794d.

36. Chaudhari N, Pereira E and Roper SD. Taste receptors for umami: the case for multiple receptors. Am J Clin Nutr. 2009;90(3): 738S-742S. doi: 10.3945/ajcn.2009.27462H.

37. Mohanty BP, Ganguly S, Mahanty A, Sankar TV, Anandan R, Chakraborty K, et al. DHA and EPA Content and Fatty Acid Profile of 39 Food Fishes from India. Biomed Res Int. 2016;8(4) :14. doi: 10.1155/2016/4027437.

38. Van Ba H, Amna T and Hwang I. Significant influence of particular unsaturated fatty acids and $\mathrm{pH}$ on the volatile compounds in meat-like model systems. Meat Sci. 2013;94(4): 480-8. doi: 10.1016/j.meatsci.2013.04.029.

39. Zhang W, Xiao S and Ahn DU. Protein oxidation: basic principles and implications for meat quality. Crit Rev Food Sci Nutr. 2013;53(11): 1191-201. doi: 10.1080/10408398.2011.577540.

40. Dermiki M, Phanphensophon N, Mottram DS and Methven L. Contributions of non-volatile and volatile compounds to the umami taste and overall flavour of shiitake mushroom extracts and their application as flavour enhancers in cooked minced meat. Food Chem. 2013;141(1): 77-83. doi: 10.1016/j.foodchem.2013.03.018.

41. Wood JD, Enser M, Fisher AV, Nute GR, Sheard PR, Richardson RI, et al. Fat deposition, fatty acid composition and meat quality: A review. Meat Sci. 2008;78(4): 343-58. doi:

10.1016/j.meatsci.2007.07.019.

42. Ulven SM and Holven KB. Metabolomic and gene expression analysis to study the effects of dietary saturated and polyunsaturated fats. Curr Opin Lipidol. 2020;31(1): 15-19. doi:

10.1097/MOL.0000000000000651.

43. Zabala-Letona A, Arruabarrena-Aristorena A, Martin-Martin N, Fernandez-Ruiz S, Sutherland JD, Clasquin $\mathrm{M}$, et al. mTORC1-dependent AMD1 regulation sustains polyamine metabolism in prostate cancer. Nature. 2017;547(7661): 109-113. doi: 10.1038/nature22964.

44. Lim HK, Rahim AB, Leo VI, Das S, Lim TC, Uemura T, et al. Polyamine Regulator AMD1 Promotes Cell Migration in Epidermal Wound Healing. J Invest Dermatol. 2018;138(12): 2653-2665. doi: 10.1016/j.jid.2018.05.029. 
45. Mackay K, Starr JR, Lawn RM and Ellsworth JL. Phosphatidylcholine hydrolysis is required for pancreatic cholesterol esterase- and phospholipase A2-facilitated cholesterol uptake into intestinal Caco-2 cells. J Biol Chem. 1997;272(20): 13380-9. doi: 10.1074/jbc.272.20.13380.

\section{Figures}
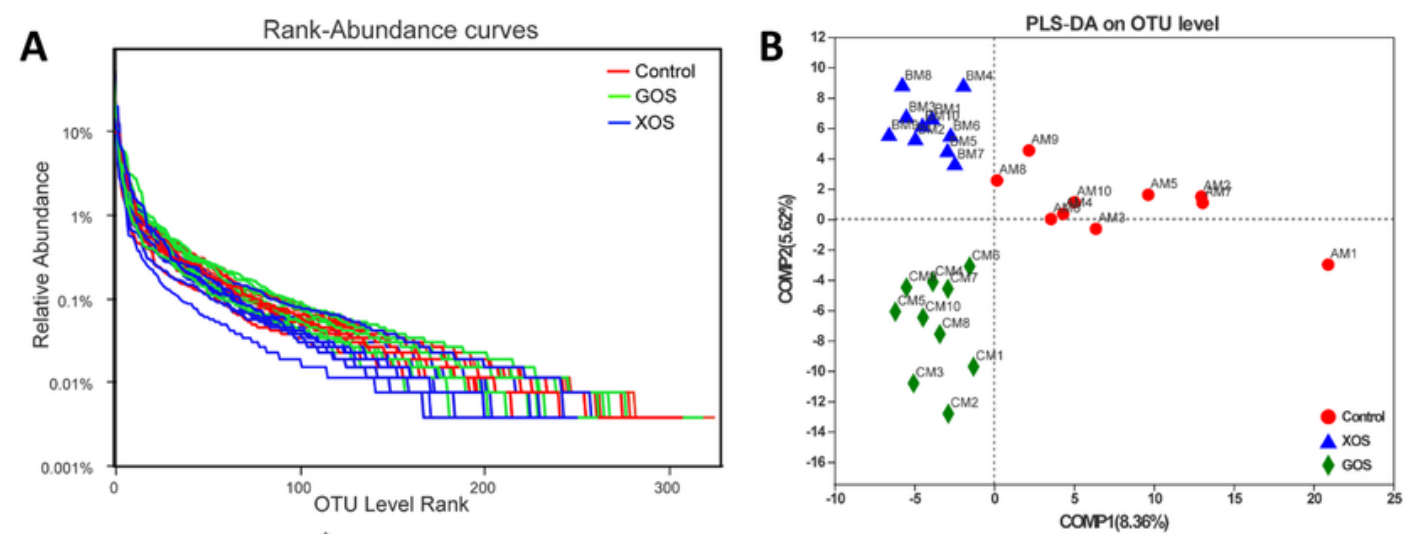

C
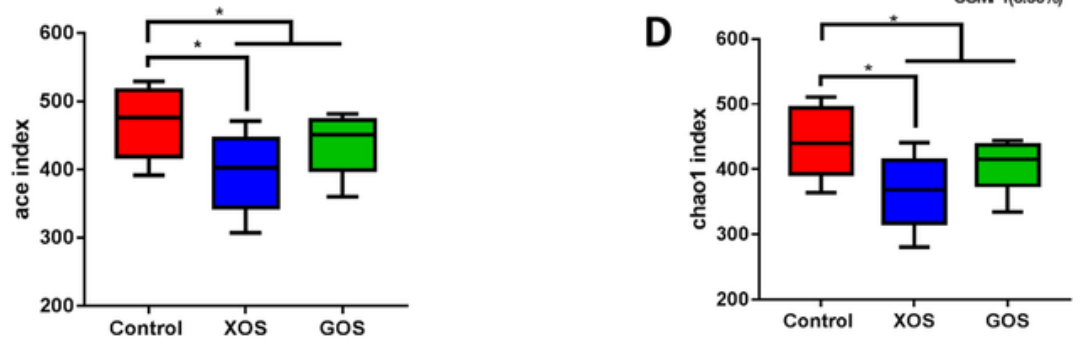

E
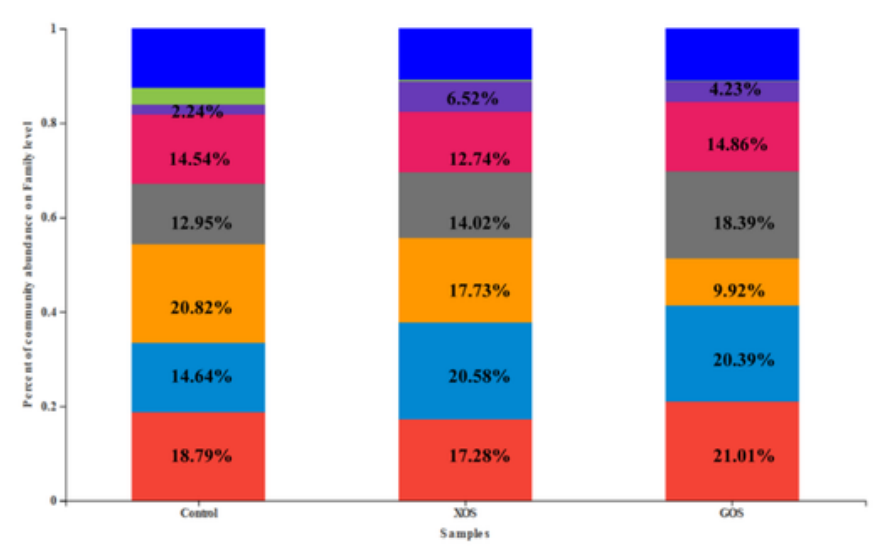

$\mathbf{F}$

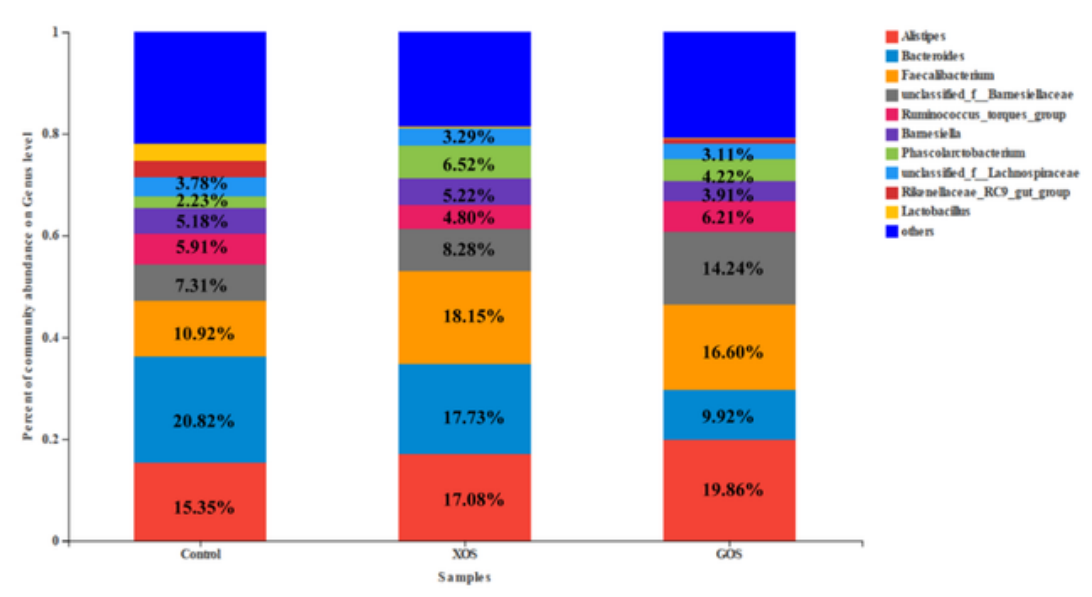

Figure 1 
Relative gut microbiota abundance at the different taxa level in the control and prebiotics groups. (A) Rank abundance curves of gut microbiota at OUT level in three groups. (B) PLS-DA analysis based on the unweighted UniFrac analysis on the OUT level. The ace (C) and chao1 (D) indx in three groups. (E-F) Relative gut microbiota abundance at the family and genus level in fecal samples from the control and prebiotics groups (GOS group and XOS group). GOS group indicated chickens was fed galactooligosaccharides (GOS); XOS group presented chickens was fed xylo-oligosaccharides (XOS).

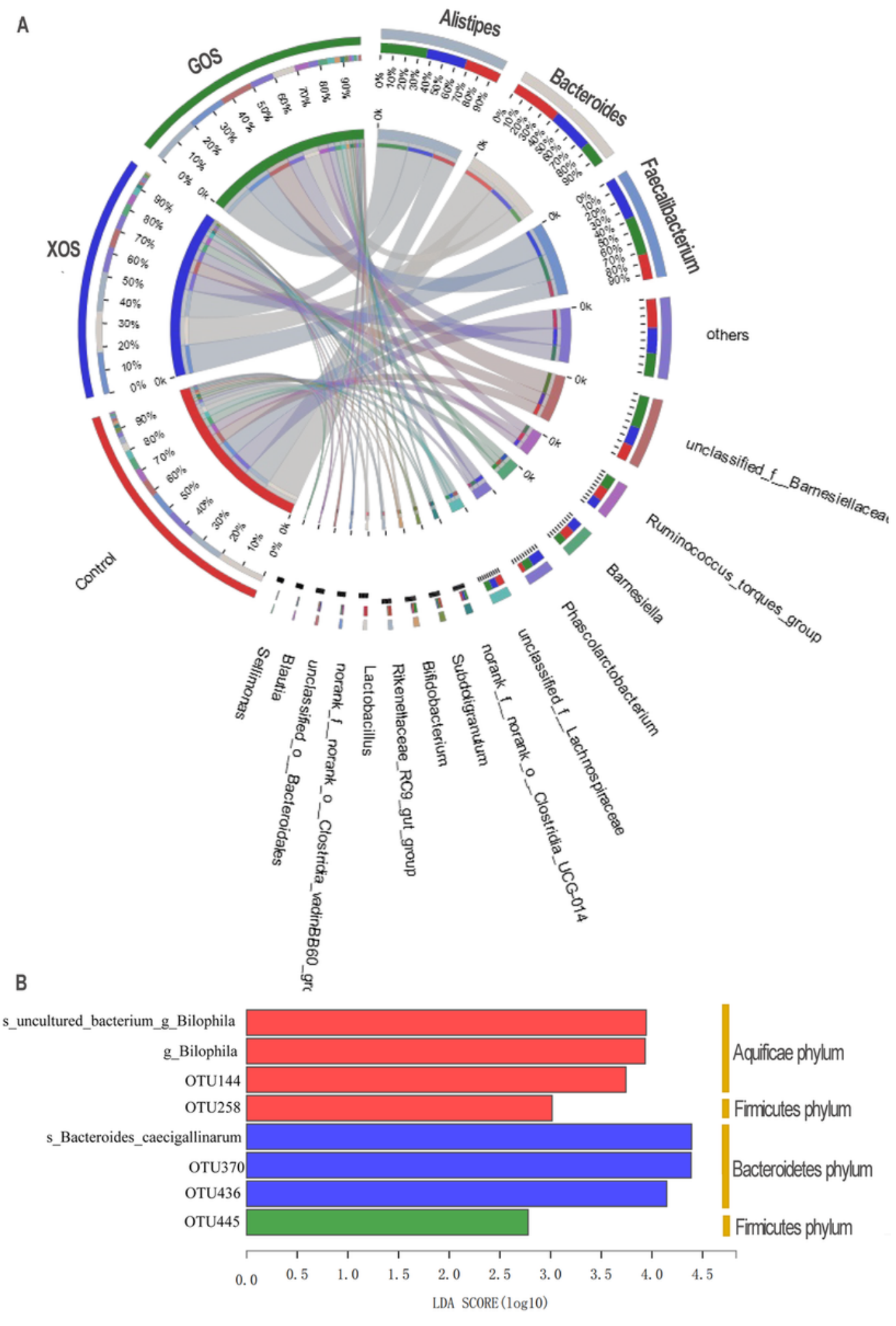

Figure 2 
The relationship and abundance of flora in three groups. (A) The relationship between flora and samples and visualized as a circos plot. (B) The differences in abundance among the three groups analyzed by LEfSe.

A

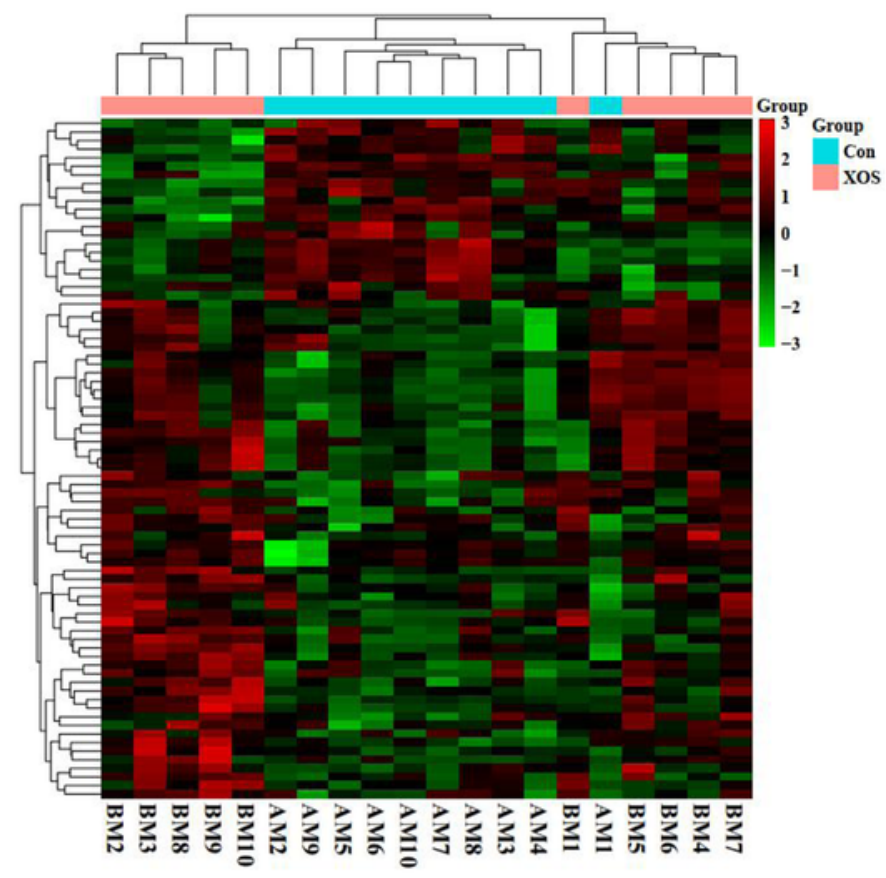

B

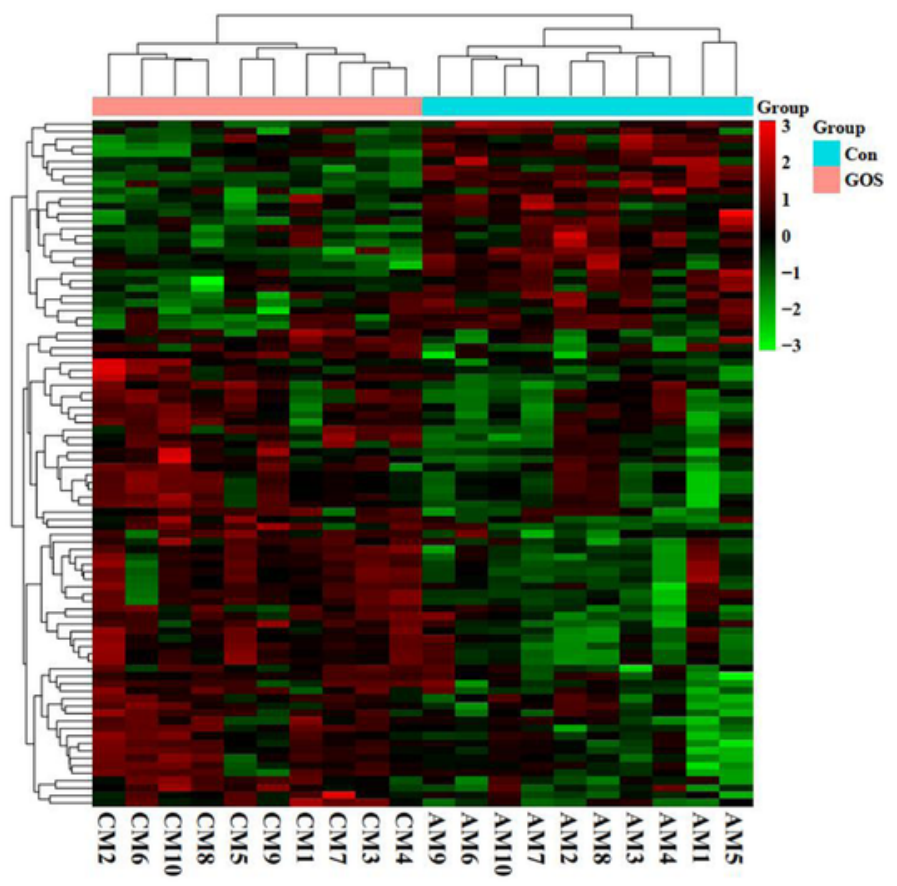

C

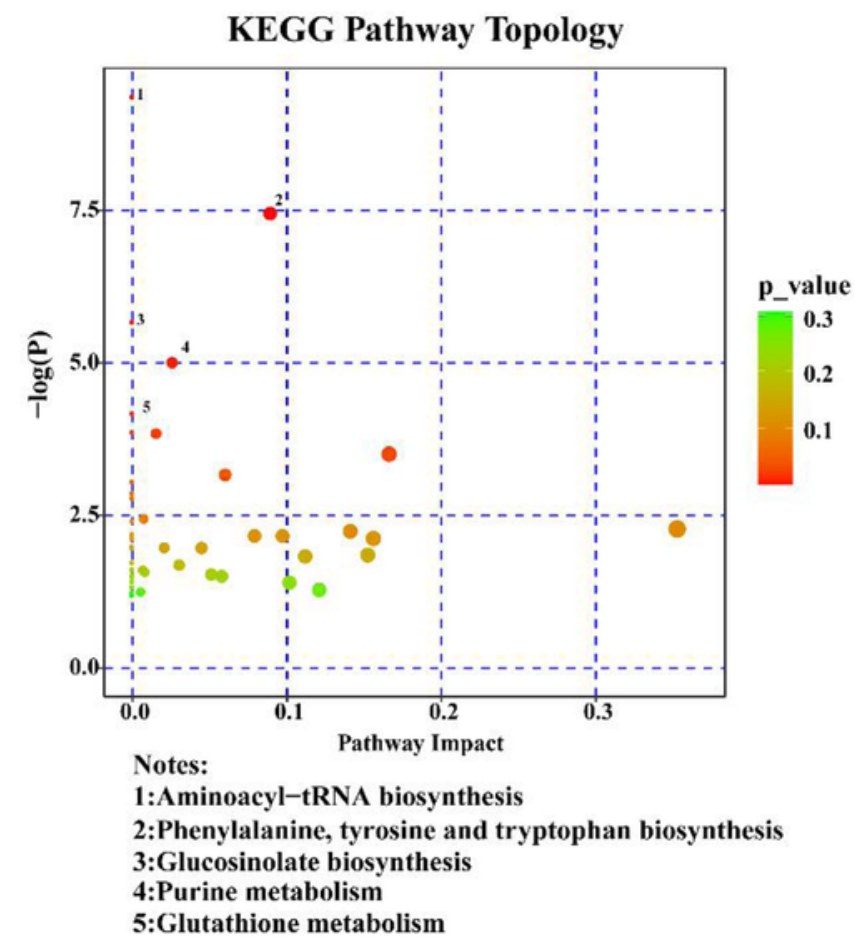

D

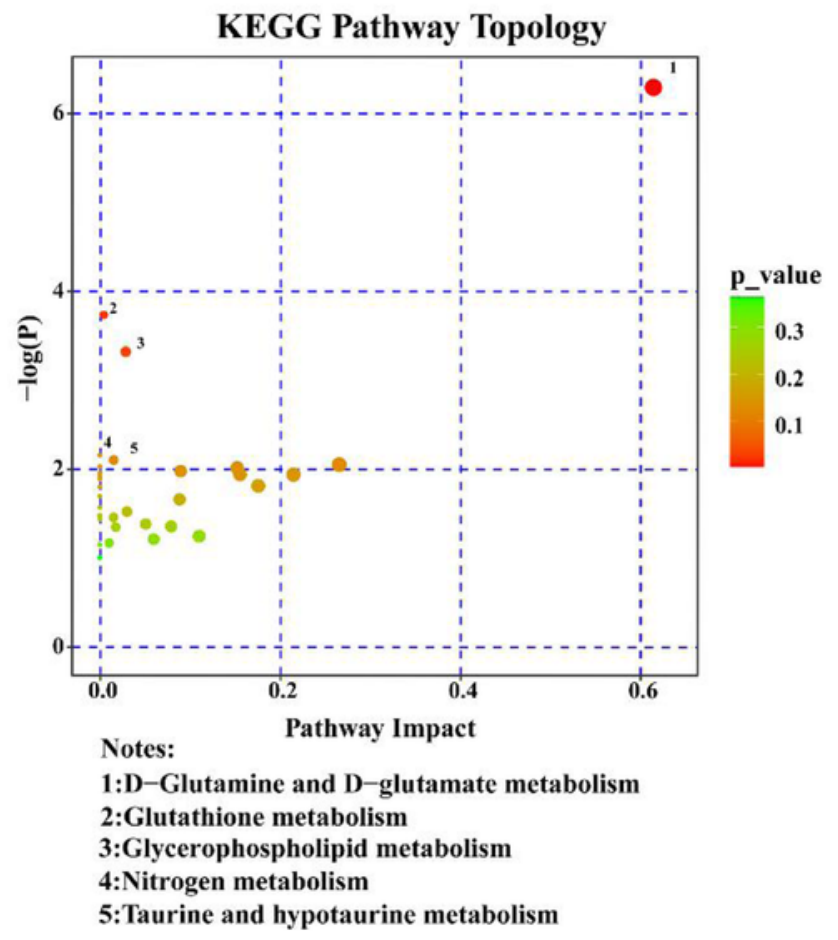

\section{Figure 3}

Common differential metabolites in the prebiotics groups compared with control group and their enriched pathways based on LC/MS data analysis. (A-B) Heatmap diagram showing fold changes of significantly 
altered metabolites in caecal contents from chickens in the prebiotics groups. Higher concentrations of metabolites are marked in red color, whereas lower concentrations of that in blue color in the XOS and GOS treatment group compared with control group. (C-D) The impact of pathways in the XOS(C) and GOS(D) groups relative to control group.

A
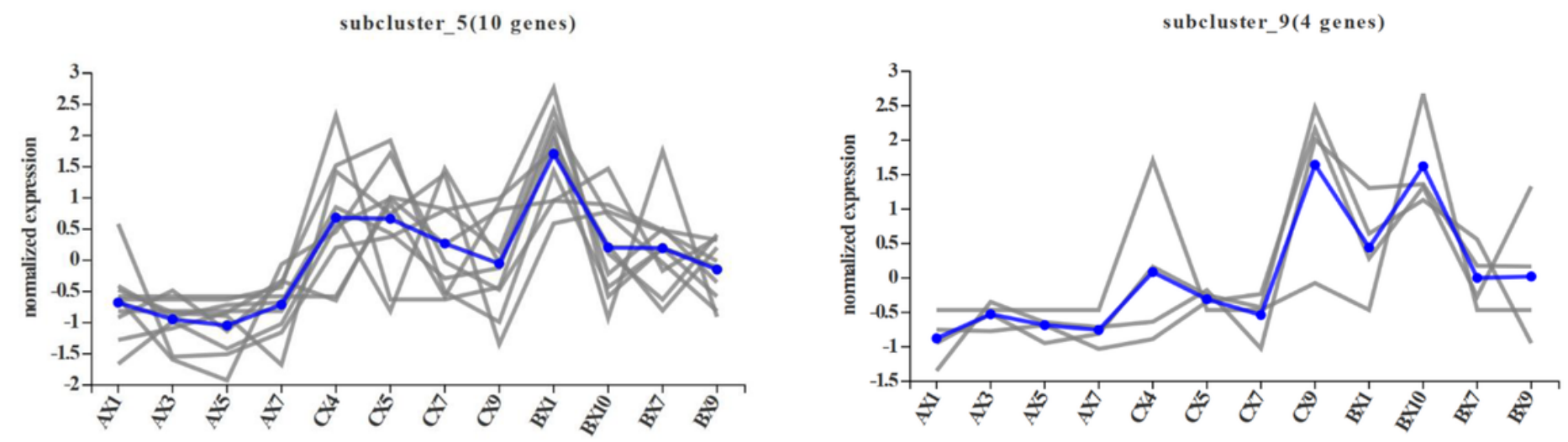

B

GO enichment analysis

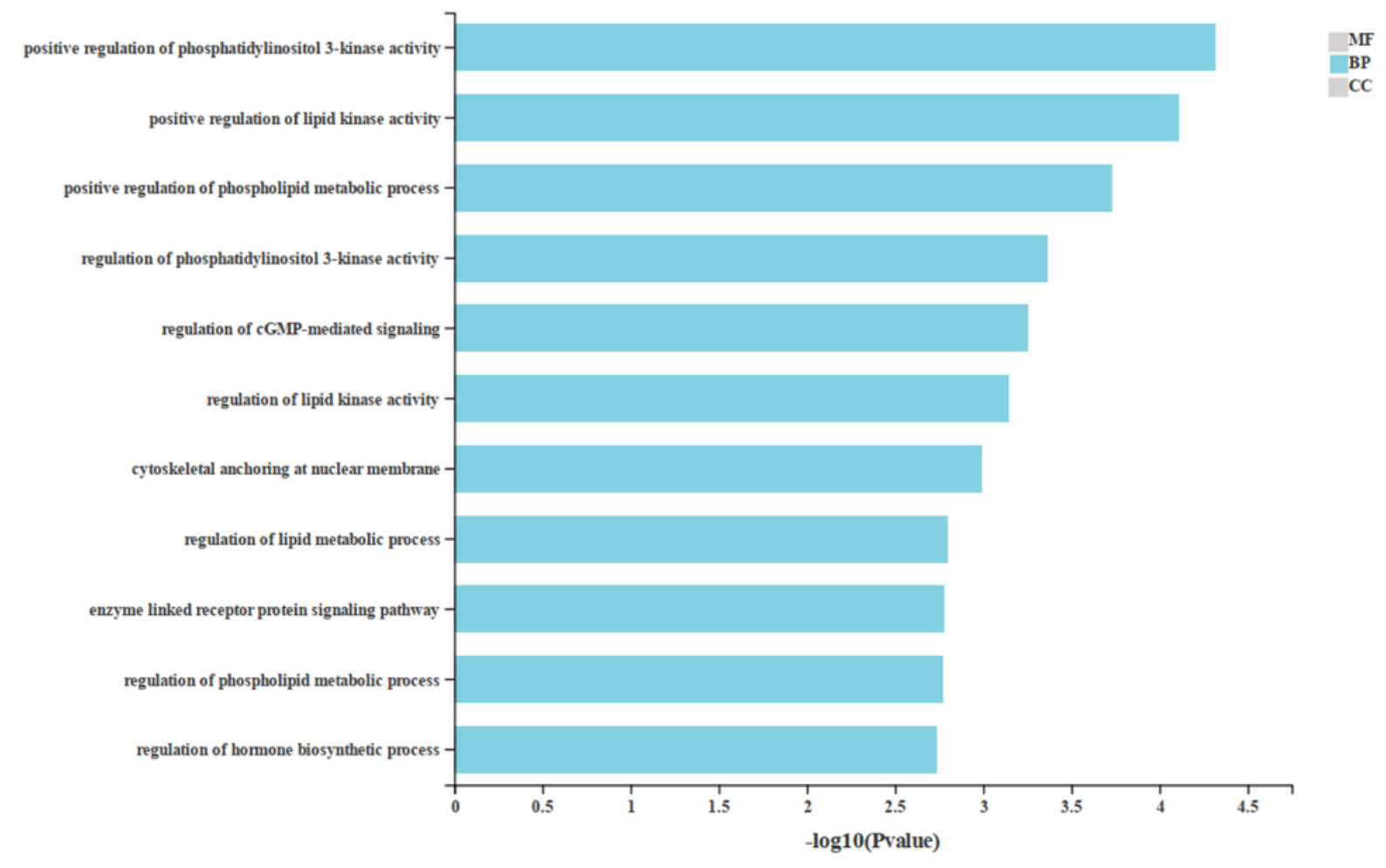

\section{Figure 4}

Subcluster and Gene ontology (GO) functional enrichment analysis in the prebiotics group via transcriptome data of chicken breast. (A-B) Subcluster analysis representing common genes with similar expression trends in each sample. Common genes differentially expressed in XOS group and GOS group compared with control group.The $x$-axis shows the sample and the $y$-axis represents the gene expression level. Each line represents the altered trend of a gene, and the blue line represents the change trend of the average expression amount in the gene set. $A X 1,3,5,7$ were the control group; $C X 4,5,7,9$ were GOS group; 
BX1,10,7,9 were XOS group. (C) Top GO biological processes for the enrichment analysis of 80 common genes. The horizontal axis indicates the -log10 ( $\mathrm{p}$-value) and the vertical axis shows the top enrichment terms in biological process. $\mathrm{P}<0.05$

A

Histogram of KEGG

Metabolism

Ogarismnl Systems

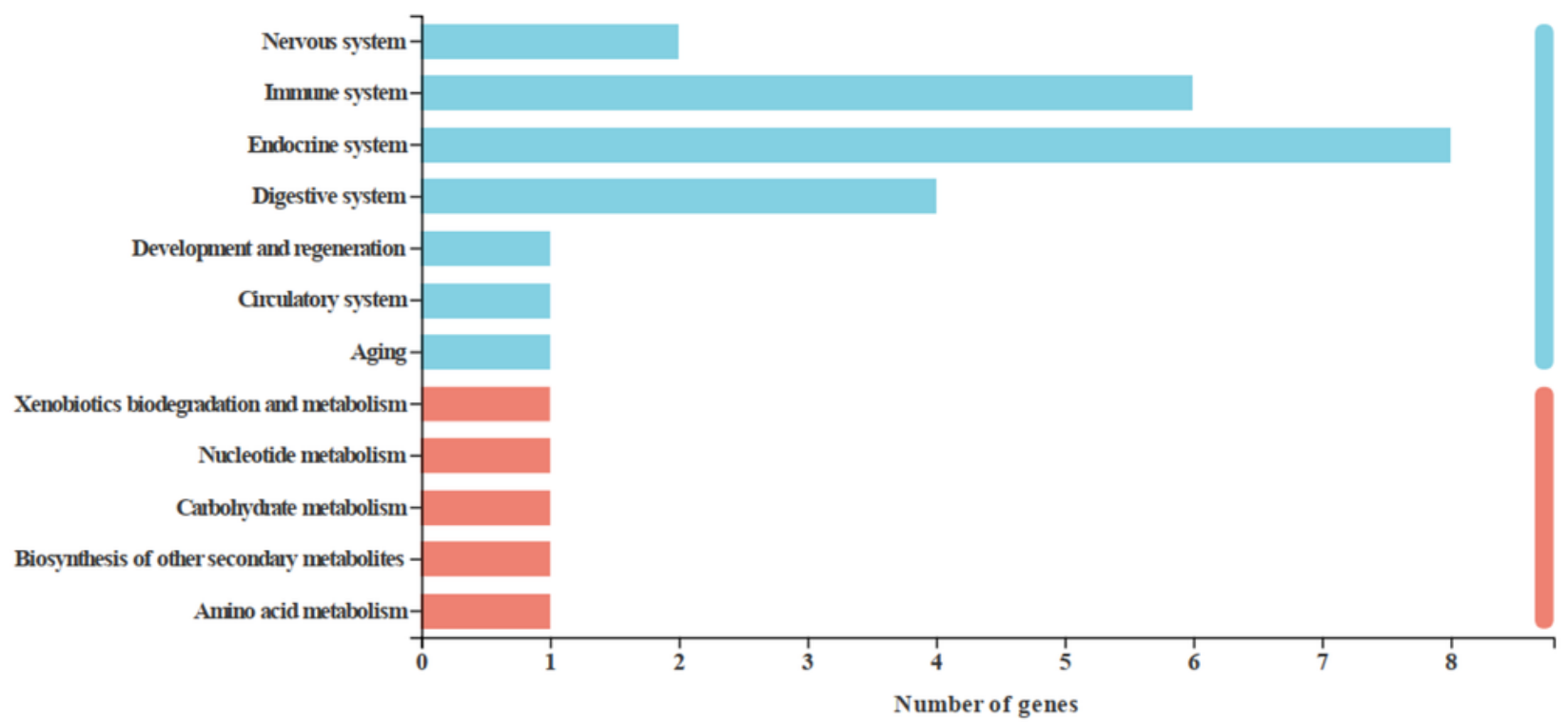

B

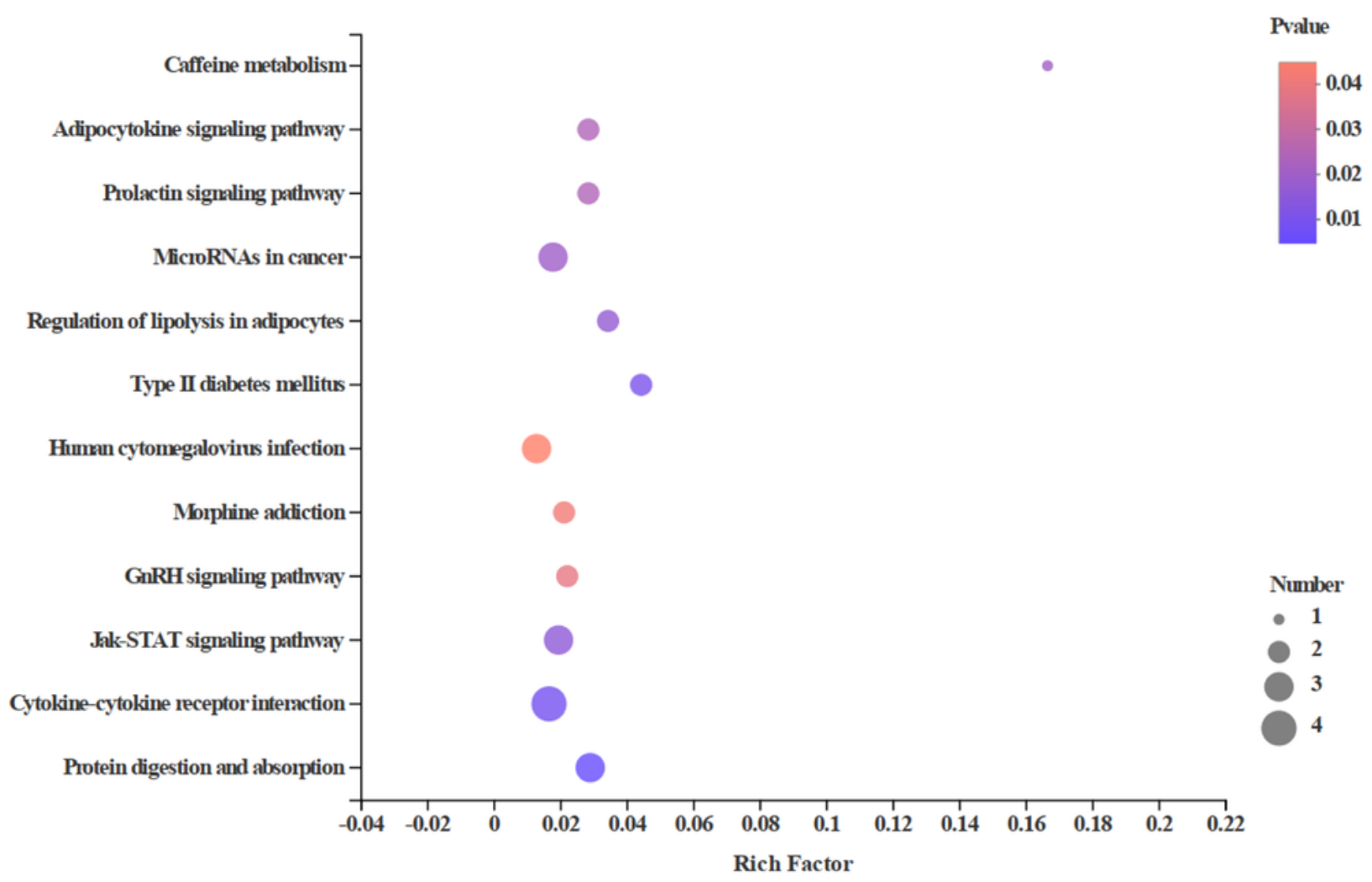

Figure 5

The Kyoto Encyclopedia of Genes and Genomes (KEGG) pathway analyses of dysregulated genes in chickens fed with XOS and GOS. (A) The most enriched pathway terms in metabolism and organismal 
systems for 80 common genes. (B) Top 12 KEGG pathways of enrichment of 80 common genes. Node color: $\mathrm{p}$ value.
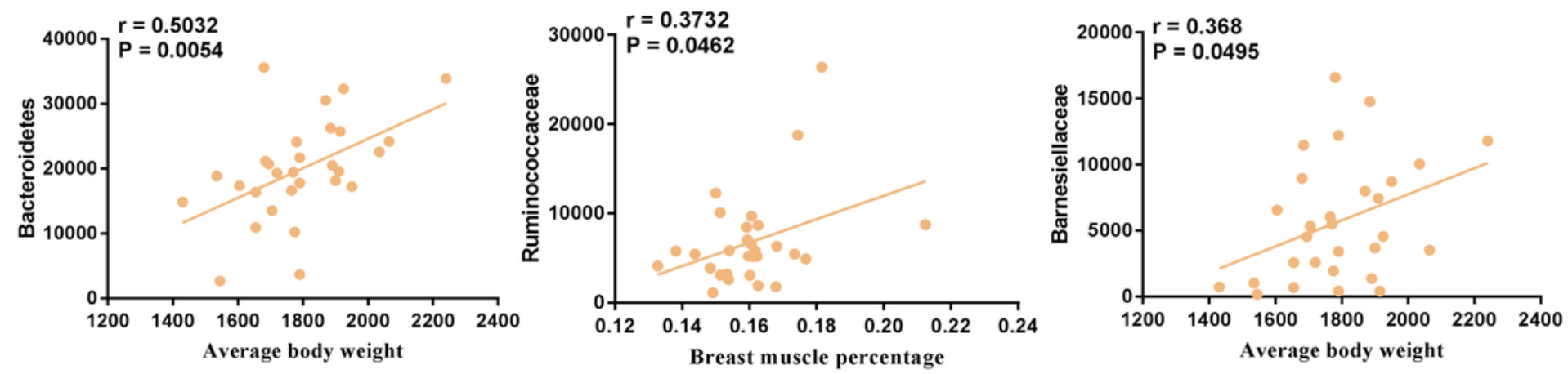

\section{Figure 6}

Caecal microbiota correlated to growth performance of broiler chickens.

A

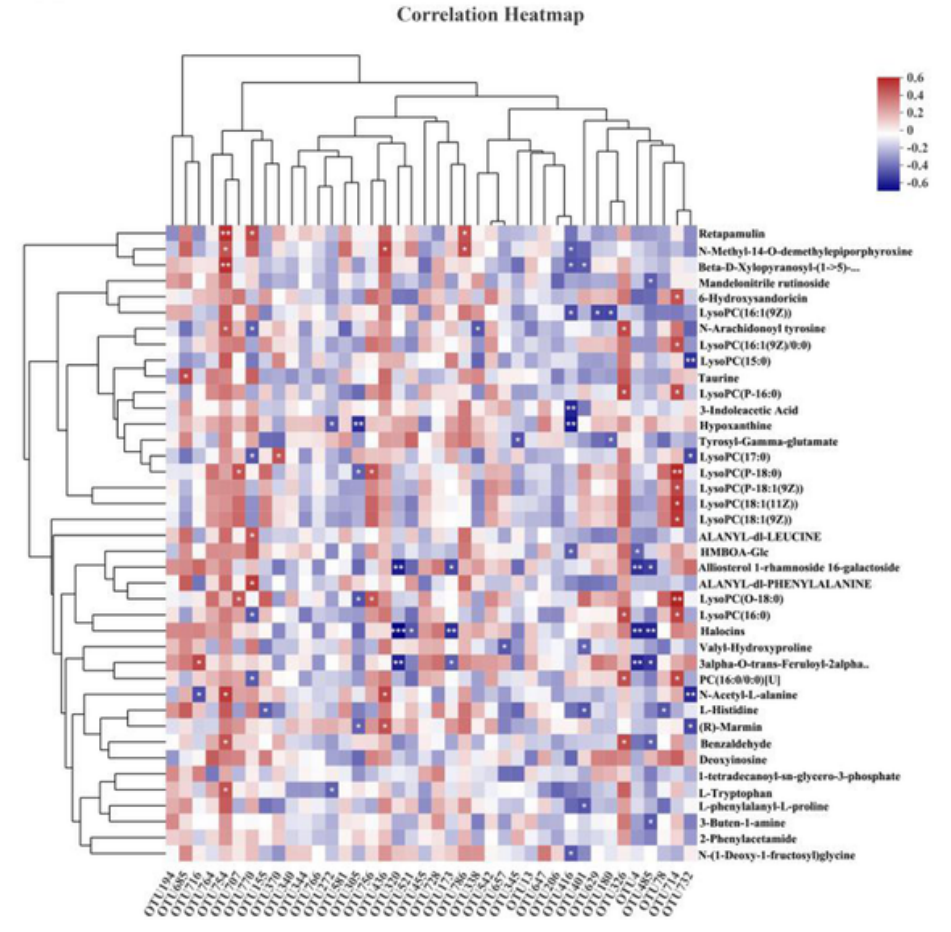

B

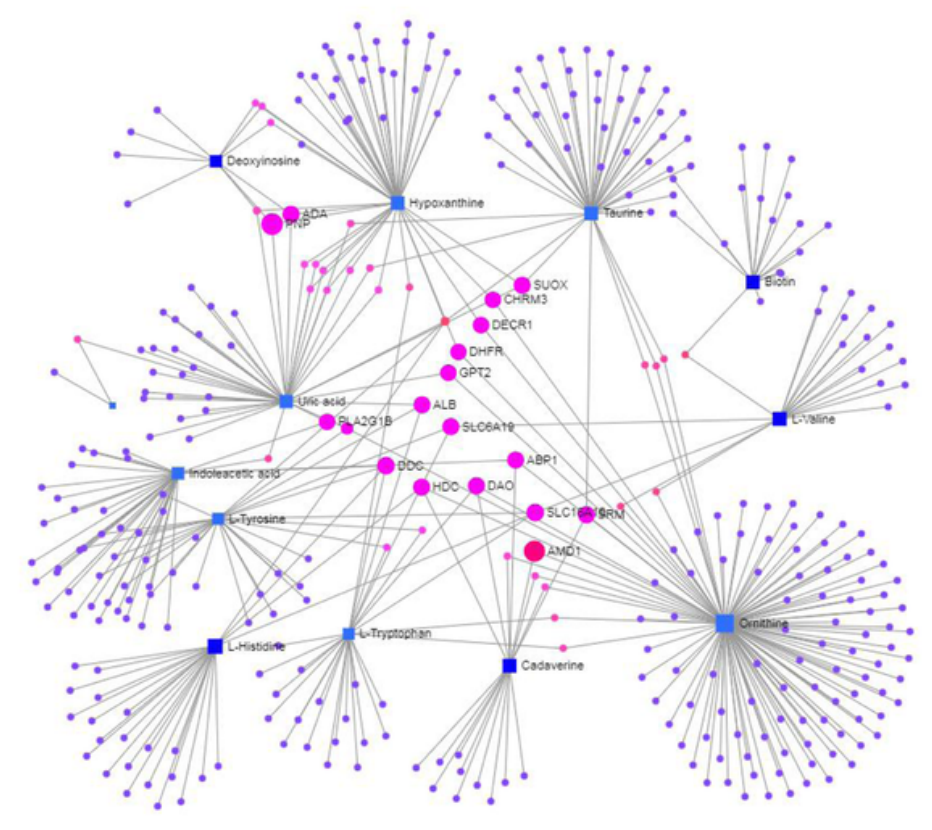

\section{Figure 7}

Correlation network of OTUs and metabolites, and interaction network of metabolites and genes. (A) Heatmap diagram depicting the correlation between the top 40 OTU and differential metabolites in XOS group. The red color indicated higher correlations, whereas The blue color indicated lower correlations. ${ }^{*} P<0.05,{ }^{*} P<0.01,{ }^{*} * P<0.001$. (B) The differential genes-metabolite interaction network analysis based on STITCH database. The red and purple circles represent the genes; the blue square represent the metabolites. 

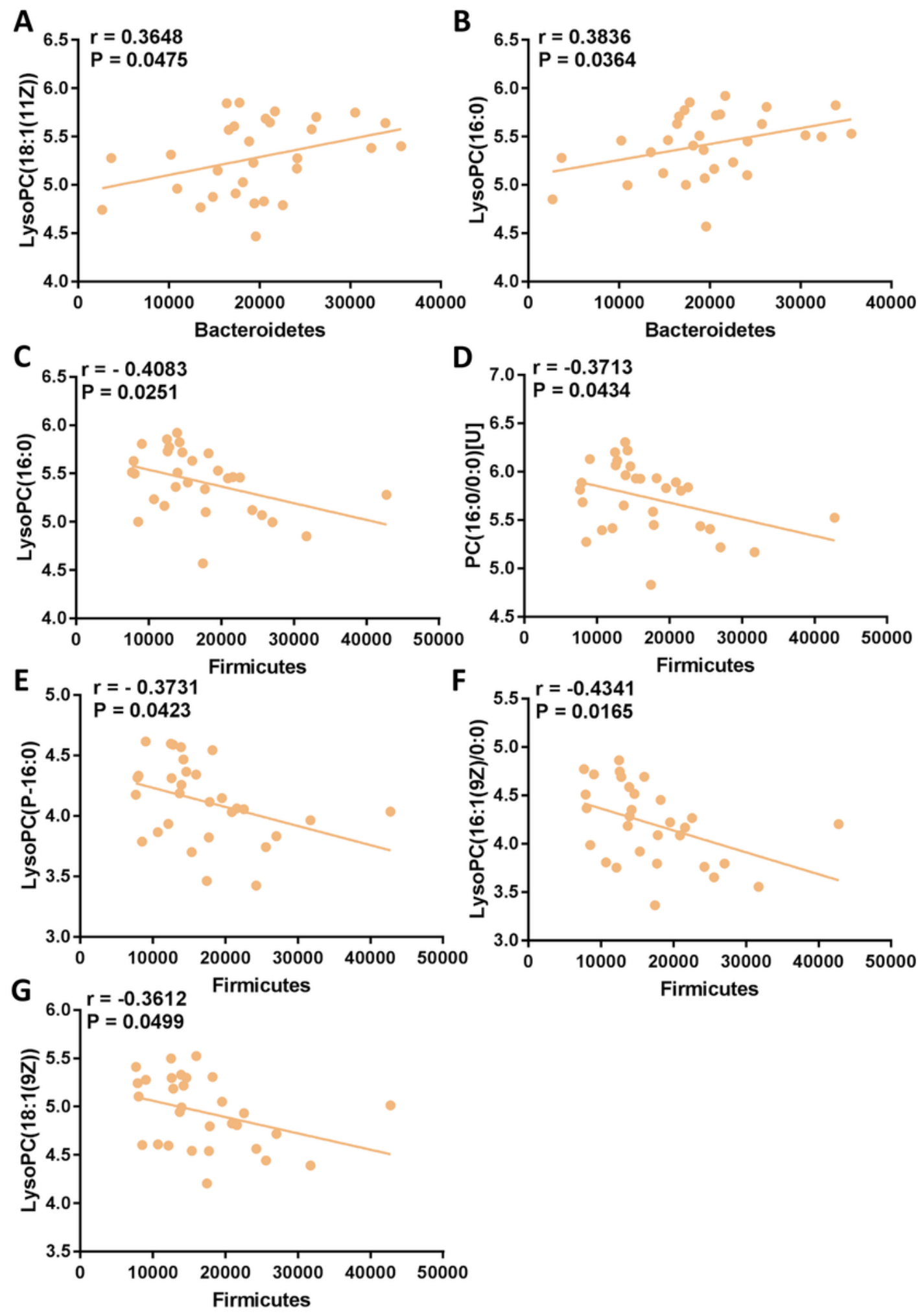

Firmicutes

Figure 8

Correlation between Bacteroidetes and Firmicutes abundance and LysoPC. (A-B) Bacteroidetes was positively correlated with LysoPC LysoPC(18:1(11Z)) and LysoPC(16:0). (C-G) Firmicutes was negatively correlated with LysoPC(16:0), LysoPC(16:1(9Z)/0:0), LysoPC(P-16:0), LysoPC(16:1(9Z)), and LysoPC(18:1(9Z)). 


\section{Supplementary Files}

This is a list of supplementary files associated with this preprint. Click to download.

- FigureS1.tif

- Figures2.tif

- TableS1.xlsx

- TableS2.xIsx

- TableS3.xIsx

- TableS4.xlsx

- TableS5.xIsx 